\title{
O discurso dos gestores sobre a equidade: um desafio para o SUS
}

\author{
The discourse of managers on equity: \\ a challenge for Brazil's Unified Health System (SUS)
}

Gabriela Ferreira Granja ${ }^{1}$

Elma Lourdes Campos Pavone Zoboli ${ }^{1}$

Lislaine Aparecida Fracolli ${ }^{1}$

${ }^{1}$ Escola de Enfermagem, Universidade de São Paulo. Av. Dr. Enéas de Carvalho Aguiar 419, Cerqueira César. 05.403-000 São Paulo SP Brasil. gabrielafgranja@gmail.com
Abstract Equity is one of the pillars of Brazil's Unified Health System (SUS). However, analysis of the scientific literature shows that the concept of equity is polysemic and open to many interpretations. The scope of this article is to identify the meanings conferred upon equity in the discourse of SUS managers. Semi-structured interviews were conducted with individuals in three municipalities in the state of Sao Paulo, namely Marília, São Carlos and Santos. The interviews were recorded, transcribed and organized using the Discourse of the Collective Subject method. In the analysis of the results, three core categories were identified: Treat everyone in an egalitarian manner; treat the unequal in an unequal manner, prioritizing the most needy in accordance with their social and economic status; and treating the unequal in an unequal manner, prioritizing specific groups in accordance with risk criteria. The managers acknowledge equity as being a SUS principle and seek prudent clinical and epidemiological criteria to justify their choices in allocating resources. There is a need for further research into the concept and operationalization of equity and the need to hear the other actors of SUS who are involved in the actions and are jointly responsible for decisions on healthcare.

Key words Equity in health, Health management, Public health policies
Resumo A equidade é um dos pilares da Sistema único de Saúde (SUS). Entretanto, a análise da literatura científica demonstra que este conceito é polissêmico e assume diversas interpretações. Este artigo tem como objetivo identificar os sentidos conferidos à equidade no discurso dos gestores do SUS. Foram realizadas entrevistas semiestruturadas com os sujeitos de três municípios do Estado de São Paulo: Marília, São Carlos e Santos. As entrevistas foram gravadas, transcritas e organizadas através do Discurso do Sujeito Coletivo. $\mathrm{Na}$ análise dos resultados, foram encontradas três categorias centrais: Tratar todos de forma igualitária; tratar os desiguais de forma desigual, priorizando os mais necessitados, segundo a condição socioeconômica; e tratar os desiguais de forma desigual, priorizando os grupos específicos, segundo critério de risco. A polissemia encontrada na literatura científica foi também encontrada nos discursos. Os gestores reconhecem a equidade como um princípio do SUS e buscam critérios clínicos e epidemiológicos prudentes que justifiquem suas escolhas na alocação de recursos. Há necessidade de novos estudos a respeito do conceito e da operacionalização da equidade e a necessidade de ouvir os demais atores do SUS, que integram as ações e são corresponsáveis pelas decisões em Saúde.

Palavras-chave Equidade em saúde, Gestão em saúde, Políticas públicas de saúde 


\section{Introdução}

Após 21 anos de existência, o Sistema Único de Saúde (SUS) tem como um de seus principais desafios a serem enfrentados a operacionalização de seus princípios norteadores: a universalidade no acesso aos serviços, a igualdade no atendimento e a integralidade na assistência.

Embora a equidade não esteja descrita na lei 8.080 da Constituição Brasileira de $1988^{1}$, que dá sustentação ao SUS, ela também tem sido interpretada por diversos especialistas em saúde pública como um princípio do SUS e um grande desafio a ser enfrentado pelos gestores. Discussão esta, que hoje está iluminada pelas dificuldades no financiamento da Saúde no mundo todo.

A literatura tem mostrado que o termo equidade tem servido a diversas interpretações, sendo estas fundadas no princípio da igualdade de oportunidades de acesso a serviços de saúde, de recursos despendidos para cada indivíduo de uma condição particular, de vida sadia ganha por unidade monetária utilizada, de possibilidade das pessoas atingirem seu potencial de vida saudável, ou atendimento desigual a pessoas desiguais; interpretações baseadas no princípio da diferença, tratamento igual para os iguais e desigual para os desiguais ${ }^{2}$. Além disso, o conceito remete à questão da justiça distributiva, dos direitos e deveres do homem/cidadão e do Estado ${ }^{3}$.

A própria Organização Mundial da Saúde estabelece que um dos objetivos dos sistemas de saúde é o alcance do nível ótimo de saúde, distribuído de forma equitativa4 ${ }^{4}$.

O debate sobre a intervenção estatal em saúde e sua atuação em termos de equidade se ampliou consideravelmente na década de 90 , frente ao aumento das desigualdades, sobretudo no sul do Planeta, tornando este um dos principais pontos de discussão nos fóruns nacionais e internacionais $^{3}$. Essas questões buscavam dar respostas às críticas dirigidas ao sistema de saúde vigente àquela época, cujo formato deixava à margem do sistema grande parte da população brasileira: os mais pobres, os que se encontravam em condições de desvantagem social e, por isso, os que talvez mais precisassem de atenção à saúde ${ }^{5}$.

Nos dias atuais a grande discussão recai sobre quais devam ser o papel e as funções do Estado, o que cabe à sociedade e o que cabe ao mercado. Esse debate no geral se traduz numa equação "mais mercado e menos Estado", "mais Estado e menos mercado", a depender da escolha feita ${ }^{6}$.

Há um relativo consenso quanto ao diagnóstico da ineficiência e da ineficácia dos gastos pú- blicos, porém os temas da equidade na saúde e da focalização têm suscitado forte polêmica. De um lado, posições que associam a focalização à perspectiva de restrição de direitos a partir da instauração de um cardápio mínimo de ações a serem desenvolvidas pelo Estado apenas para a população mais pobre. De outro, encontram-se algumas análises que salientam a possibilidade da focalização constituir-se alternativa atraente para fazer face ao quadro de extrema pobreza e desigualdades sociais no Brasil, na medida em que estabelece prioridades de acesso dos segmentos mais vulneráveis nos programas sociais ${ }^{7}$.

A abertura da economia, a integração do país nos circuitos globalizados, o primado da economia sobre a política, do qual derivam as pragmáticas fórmulas de Estado e políticas sociais mínimas, desnatura a própria essência do SUS universal. Grande parte dos gastos públicos foi cortada em nome do ajuste econômico, o que levou a precarização do sistema e a focalização de suas ações para a população sem condições de pagar um plano privado ${ }^{8}$.

$\mathrm{Na}$ Saúde, alocar recursos financeiros ou físicos, de maneira mais eficiente e equitativa, é um desafio que o gestor e os profissionais enfrentam cotidianamente. Isso se aplica tanto no nível da macrogestão, quanto da microgestão da saúde. Ou dito de outra forma, tanto nas decisões para distribuição de recursos entre estados, regiões e municípios, programas e ações de saúde, quanto na opção clínica em solicitar ou não, para um indivíduo, a realização de determinado procedimento de diagnose ou terapia ${ }^{4}$.

Entretanto, a sociedade moderna, moralmente pluralista, carece de parâmetros e critérios objetivos para definir os significados práticos do "bom", da "vida boa", de "justiça" e assim estabelecer os limites e as exigências éticas, e este é o desafio presente para se tomar decisões na priorização de serviços e ações de saúde ${ }^{9}$.

A discussão do conceito de equidade tornase, desta forma, fundamental para os gestores do SUS, responsáveis pela alocação de recursos na Saúde nesta realidade complexa, em tempos de escassez de recursos e pluralidade moral.

Assim, o objetivo deste estudo é identificar os sentidos conferidos à equidade no discurso dos gestores do SUS.

\section{Método}

Este estudo traz uma abordagem qualitativa da equidade em saúde, pois o fenômeno investiga- 
do situa-se no universo dos significados, motivações, aspirações, crenças, valores e atitudes, que não se reduzem às abordagens quantitativas. Segundo Minayo, é a pesquisa qualitativa que permite incorporar a questão do significado e da intencionalidade como inerentes aos atos, às relações e às estruturas sociais. Assim, esta pesquisa se configura como um estudo qualitativo, de cunho descritivo e analítico ${ }^{10}$.

Fizeram parte do estudo, os municípios de Marília, São Carlos e Santos, todos localizados no Estado de São Paulo, com mais de 150.000 habitantes, em gestão plena do SUS e com diferentes Índices de Desenvolvimento Humano do Estado de São Paulo (IPRS), segundo a Fundação SEADE ${ }^{11}$.

Os sujeitos da pesquisa foram os gestores do sistema de saúde local. Limitou-se a inclusão dos gestores vinculados à gestão da atenção básica, nos diferentes níveis da administração municipal: central, regional e local. A escolha destes gestores foi feita de acordo com a realidade de cada município. Optou-se por focar a atenção básica por representar o nível de acesso do cidadão ao Sistema de Saúde.

Outro critério decisivo para inclusão foi o consentimento livre e esclarecido por parte dos sujeitos de pesquisa, o que nos remete à observância das diretrizes para ética em pesquisa no desenvolvimento dos trabalhos.

Foram realizadas entrevistas semiestruturadas com as seguintes questões: Fale-me sobre a equidade na Atenção à Saúde. Como você vê isso na sua prática? Como você define equidade?

Todos os depoimentos coletados foram gravados, em meio digital, e transcritos. Os dados coletados nas entrevistas foram organizados por meio do Software do Discurso do Sujeito Coletivo (DSC), com vistas a identificar os sentidos conferidos à equidade, um dos pilares do SUS ${ }^{12}$.

O DSC constitui uma técnica de organização de dados discursivos em pesquisa qualitativa que permite resgatar o estoque de representações que se tem sobre um determinado tema em um dado universo. Os discursos são submetidos a uma análise de conteúdo que se inicia com a decomposição destes nas principais ideias centrais presentes em cada um individualmente e em todos reunidos, seguindo-se a uma síntese que visa à reconstituição discursiva da representação social ${ }^{12}$.

$\mathrm{Na}$ organização dos dados, as questões realizadas nas entrevistas foram agregadas em uma única, de forma que os discursos dos gestores do SUS respondessem, essencialmente, a seguinte questão norteadora: "O que é equidade?"
Segundo determinações da Resolução CNS/ MS $196 / 96^{13}$, que regulamenta a ética da pesquisa envolvendo seres humanos no Brasil, este estudo foi aprovado pelo Comitê de Ética em Pesquisa da Escola de Enfermagem da USP e foi submetido aos Comitês de ética das Prefeituras Municipais.

\section{Resultados e discussão}

Em relação à questão norteadora: "O que é equidade?", encontramos nos discursos dos gestores dos três municípios duas ideias centrais, que deram origem a duas categorias centrais e seus respectivos discursos.

\section{A- Tratar todos de forma igualitária}

Atender as pessoas que procuram o SUS da mesma forma, dar um serviço que seja igual ou semelhante a todos aqueles que buscam o SUS, com dignidade, com respeito e conseguir oferecer aquilo a todos os cidadãos da mesma forma, sem tratálos com diferença. OSUS é universal, tem que atender todo mundo igualmente, independente de raça, sexo ou condição social.

Neste discurso é possível identificar a presença marcante da igualdade e da universalidade do acesso como princípios reconhecidos pelos gestores. Ao recordarmos que apenas uma parcela da população tinha direito à saúde até a promulgação da Constituição de 1988, a saúde como um direito universal e igualitário, representou um grande avanço para as Políticas de Saúde no Brasil.

A identidade das pessoas conta em nossos dias e em nossas sociedades com um componente irrenunciável, a igualdade de todos os cidadãos em dignidade ${ }^{14}$.

$\mathrm{Na}$ análise dos relatórios oficiais das Conferências Nacionais de Saúde observou-se uma tendência de equidade ser compreendida como igualdade. Os autores desta pesquisa apontam que as discussões não avançam para os grupos em desvantagem ${ }^{15}$.

A respeito da relação entre os conceitos de justiça social e equidade, John Rawls ${ }^{16}$ afirma que cada pessoa tem o mesmo direito irrevogável a um esquema plenamente adequado de liberdades básicas iguais que seja compatível com o mesmo esquema de liberdades para todos (princípio da igualdade). Além disso, acrescenta que desigualdades sociais e econômicas devem satisfazer duas condições: primeiro devem estar vinculadas a cargos e posições acessíveis a todos em 
condições de igualdade equitativa de oportunidades (igualdade de oportunidades); e em segundo lugar, têm de beneficiar ao máximo os membros menos favorecidos da sociedade (princípio da diferença). O segundo princípio (da diferença) só poderá ser aplicado se o princípio anterior (da igualdade) for plenamente satisfeito, sendo que esta mesma ordem lexical vale para a igualdade de oportunidades ${ }^{16}$.

A segunda categoria foi desdobrada em duas subcategorias para melhor retratar os critérios identificados nos discursos para a delimitação do que são os mais necessitados, como é mostrado a seguir:

\section{B1- Tratar os desiguais de forma desigual, priorizando os mais necessitados segundo a condição socioeconômica}

Equidade é diferente de igualdade. Tratar os desiguais de forma desigual. Administrar de tal forma que todos tenham diante de sua expectativa o melhor que o sistema de saúde pode oferecer, diferentes situações, com diferentes acessos. É você dar o justo, aquilo que o usuário realmente precisa e não o desnecessário. A equidade é definir normas na organização dos serviços, esse é o papel do gestor cotidianamente, priorizar uma ação de saúde, construir políticas públicas focalizadas porque não existe recurso para tudo, mas quem tem mais necessidade. Priorizar as pessoas que são mais vulneráveis a determinada doença, que moram em favela, que são realmente SUS dependente, diferente de quem utiliza um convênio e usa a Atenção Básica somente nas campanhas de vacina. As pessoas que mais necessitam do serviço público de saúde devem ter um acesso mais facilitado, considerando o perfil epidemiológico das populações mais periféricas, mais carentes, os indivíduos menos incluídos socialmente. Se você tratar todos igualmente na questão econômica, você vai perpetuar a desigualdade, pois desta forma quem tem muito vai continuar tendo muito e quem tem pouco vai continuar tendo pouco. É o papel do gestor, identificar aqueles que são mais dependentes, dar uma atenção mais completa, ter um sistema mais acolhedor.

Neste discurso, o sentido de equidade está relacionado à priorização dos recursos na Saúde em benefício dos grupos sociais que se encontram em desvantagem econômica, em especial àqueles indivíduos que não tem acesso a um plano de assistência à saúde privado.

A Organização Mundial da Saúde (OMS), no texto de Margareth Whitehead, de 1990, apresenta a evidência de que grupos desfavorecidos, não só sofrem maior incidência de doenças, mas também as têm mais crônicas e incapacidades em idade precoce. Ressalta que o termo equidade tem uma dimensão ética e moral e refere-se à ausência de diferenças evitáveis e injustas, determinadas histórico e socialmente ${ }^{17}$.

\section{B2- Tratar os desiguais de forma desigual, priorizando os grupos específicos, segundo critério de risco}

Tem que ter um olhar específico, uma atenção especial no sentido de ver qual é a necessidade maior. As mulheres têm mais vulnerabilidade em comparação aos homens, como por exemplo, as mulheres negras e as profissionais do sexo, que vivem em situação de desigualdade. É planejar as ações em cima das prioridades, saúde materno-infantil, gravidez na adolescência, idoso. Os indicadores vão mostrando se estamos no caminho certo ou não para propor ações que respondam a essas situações de maior risco, organizando um cuidado diferente.

Neste discurso, os gestores demonstram uma busca por critérios objetivos na definição da alocação de recursos em saúde, segundo risco de adoecimento de alguns grupos específicos.

Na definição dos grupos específicos, alguns critérios baseados em objetividade médica apontam para a eficácia clínica, a possibilidade de ação preventiva, a vulnerabilidade da doença ou do agravo à tecnologia e ao saber científico disponível ${ }^{18}$.

Uma corrente de pensamento, bastante difundida pelos planejadores do setor de saúde, é o utilitarismo. Para eles, o correto, o justo, é a alocação de recursos que proporciona maior benefício, mais saúde, para um maior número de pessoas. Instrumentos de planejamento como custo/efetividade baseiam-se na noção de maximização dos benefícios ${ }^{19}$.

Para os que defendem a justa distribuição equitativa, o utilitarismo poderia justificar o sacrifício de pequenos interesses, expressos numericamente, por interesses mais prevalentes, podendo resultar em práticas injustas ou mesmo discriminatórias contra grupos minoritários.

$\mathrm{Na}$ análise da literatura científica, são propostos alguns caminhos para superar as dificuldades de operacionalização da equidade em saúde: a epidemiologia, como conhecimento imprescindível à compreensão dos determinantes e condicionantes do processo saúde-doença; a valorização da experiência cotidiana de profissionais e usuários na definição de prioridades na saúde e o redimensionamento das ações para o nível local na busca da equidade e da atenção integral ${ }^{20}$. 
Os critérios epidemiológicos se adéquam estritamente para apontar necessidades de atenção à saúde segundo parâmetros da morbi-mortalidade populacionais, ou para a definição da situação de saúde da população, e não como critério de escolha entre duas ou mais situações para o estabelecimento de prioridades em uma localizada alocação de recursos. Estas dependem, sobretudo, de diretrizes prévias que devem ser estabelecidas por um processo de negociação política que implique a representação de interesses das distintas esferas de governo ${ }^{21}$.

Em estudo realizado no Rio Grande do Sul, no período de 1995 a 2001, foi possível constatar que a aplicação de recursos na saúde tem se concentrado nos grandes aglomerados urbanos e que os critérios de utilização destes têm beneficiado os municípios de menor Índice de Desenvolvimento Humano ${ }^{22}$.

$\mathrm{Na}$ agenda atual do Setor Saúde, o princípio da equidade encontra-se condicionado aos objetivos de eficiência, submetido aos "limites de caixa” e de custo-efetividade que, por sua vez, não inclui a análise dos objetivos de determinada política e excluem explicitamente, a valoração dos fins que a justificam. As ações de saúde aos grupos específicos ficam reduzidas às políticas de focalização (para os mais pobres) e de privatização ${ }^{3}$.

É preciso estar alerta, pois as discussões sobre recursos em saúde têm se restringido aos aspectos monetários, e a necessidade de identificar o que é prioritário e distribuir equitativamente os recursos tem sido substituída pela ideia do racionamento, não por meio de prioridades e inclusão universal, mas sim por exclusão seletiva ${ }^{23}$.

Seria necessário retomar a discussão das políticas sociais numa perspectiva de metapolítica, que significa revigorar a discussão dos princípios e valores que lhe são subjacentes e definir princípios de justiça coerentes e consistentes nos quais se apoiar, além de operacionalizá-los de forma efetiva. Portanto, falta incluir a negociação polí- tica do cálculo de consenso possível e de dissenso suportável, ou em última instância, o grau de desigualdade suportável para uma determinada sociedade ${ }^{3}$.

Torna-se necessário buscar novas formas de relação entre Estado e sociedade que promovam a autonomia dos sujeitos sociais para praticarem suas próprias escolhas, em detrimento de simplesmente reforçar a redução dos sujeitos sociais a condição de cidadãos consumidores dos serviços providos pelo Estado ${ }^{23}$.

\section{Considerações finais}

Este estudo aponta a necessidade de novas pesquisas a respeito do conceito e da operacionalização da equidade, reconhecida pelos planejadores do SUS, como pilar da Política Pública de Saúde no Brasil.

A efetivação da equidade como um princípio é um desafio para os atores do SUS. Aos gestores, torna-se necessário questionar se a distribuição de bens como habitação, assistência à saúde, educação e outros requer uma distribuição igualitária dos recursos ou pressupõe privilegiar determinados indivíduos ou grupos sociais. Além disso, seria fundamental questionar quais critérios seriam necessários para estabelecer essa distribuição.

Os discursos dos gestores e a análise da literatura científica demonstram a existência de diferentes valores éticos que podem determinar as decisões na alocação de recursos em saúde.

Essa diversidade de sentidos para equidade é fruto da realidade complexa que vivemos e, portanto, a equidade deve ser analisada como objeto complexo e dinâmico, em que todos os atores envolvidos, profissionais pesquisadores e usuários do SUS devem ser ouvidos e considerados no planejamento das ações em saúde.

\section{Colaboradores}

GF Granja, ELCP Zoboli e LA Francolli participaram igualmente de todas as etapas de elaboração do artigo. 


\section{Referências}

1. Brasil. Constituição Federal. Título VIII. Da Ordem Social; capítulo II-seção I, art. 194, de 1988. Diário Oficial da União 1988; 5 out.

2. Fortes PAC. Reflexões sobre o princípio ético da justiça distributiva aplicado aos sistemas de saúde. In: Fortes PAC, Zoboli ELCP, organizadores. Bioética e Saúde Pública. São Paulo: Edições Loyola; 2003. p.35-47.

3. Almeida C. Equidade e reforma setorial na América Latina: um debate necessário. Cad Saude Publica 2002; 18(Supl):23-36.

4. Brasil. Conselho Nacional de Secretários de Saúde (CONASS). Vigilância em Saúde. Brasília: CONASS; 2007. (Coleção Progestores - para entender a gestão do SUS, V. 6).

5. Viana ALA, Fausto MCR, Lima LD. Política de Saúde e Equidade. São Paulo Perspectiva 2003; 17(1):58-68.

6. Cohn A. O estudo das políticas de saúde: implicações e fatos. In: Campos GWS, Minayo MCS, Akerman M, Drumond Júnior M, Carvalho YM, organizadores. Tratado de Saúde Coletiva. São Paulo/Rio de Janeiro: Hucitec, Fiocruz; 2006. p. 231-258.

7. Senna MCM. Equidade e Política de Saúde: algumas reflexões sobre o Programa Saúde da Família. Cad Saude Publica 2002; 18(Supl.):203-211.

8. Braga IF. A sociedade civil e as Políticas de Saúde no Brasil do anos 80 à primeira década do século XXI. In: Morosini MV, organizador. Sociedade, Estado e direito à saúde. Rio de Janeiro: Fiocruz; 2007.

9. Fortes PAC. O dilema bioético de selecionar quem deve viver: um estudo de microalocação de recursos escassos em saúde [tese]. São Paulo: Universidade de São Paulo; 2000.

10. Minayo MCS. O desafio do conhecimento: pesquisa qualitativa em saúde. $4^{\text {a }}$ Edição. São Paulo, Rio de Janeiro: Hucitec, Abrasco; 1996.

11. Fundação Sistema Estadual de Análise de Dados (SEADE). Índice Paulista de Responsabilidade Social (IPRS), versão 2012. Secretaria de Planejamento e desenvolvimento Regional do Estado de São Paulo, 2012. [acessado 2013 set 30]. Disponível em: http:/ /www.iprsipvs.seade.gov.br/view/index.php?selLoc $=0 \&$ selTpLoc $=2 \&$ prodCod $=1$

12. Lefèvre F, Lefèvre AMC, Teixeira JJV, organizadores. O Discurso do Sujeito Coletivo: uma nova abordagem metodológica na pesquisa qualitativa. Caxias do Sul: EDUCS; 2000.
13. Brasil. Ministério da Saúde (MS). Conselho Nacional de Saúde. Resolução no 196 de 10 de outubro de 1996. Diretrizes e Normas Regulamentadoras de Pesquisas Envolvendo Seres Humanos. Diário Oficial da União 1996; 16 out.

14. Cortina A. Alianza y Contrato. Política, Ética y Religión. Madrid: Editorial Trotta; 2005.

15. Pinheiro MC, Westphal MF, Akerman M. Eqüidade em saúde nos relatórios das conferências nacionais de saúde pós-Constituição Federal brasileira de 1988. Cad Saude Publica 2005; 21(2):449-458.

16. Rawls J. Justiça como equidade: uma reformulação. São Paulo: Martins Fontes; 2003.

17. Luiz OC. Direito e equidade: princípios éticos para a saúde. Arq. Méd. ABC 2005; 30(2):69-75.

18. Fortes PAC. Reflexões sobre o princípio ético da justiça distributiva aplicado aos sistemas de saúde. In: Fortes PAC, Zoboli ELCP, organizadores. Bioética e Saúde Pública. São Paulo: Edições Loyola; 2003. p.35-47.

19. Lucchese PTR. Equidade na gestão descentralizada do SUS: desafios para a redução de desigualdades em saúde. Cien Saude Colet 2003; 8(2):439-448.

20. Elias PE. A utilização da noção de equidade na alocação de recursos em tempos de pensamento (neo) liberal: anotações para o debate. Cien Saude Colet 2005; 10(2):289-292.

21. Pelegrini MLM, Castro JD, Draccher ML. Equidade na alocação de recursos para a saúde: a experiência do Estado do Rio Grande do Sul. Cien Saude Colet 2005; 10(2):275-286.

22. Fortes PAC, Zoboli, ELCP. Os princípios do Sistema Único de Saúde: potencializando a inclusão social na atenção saúde. $O$ mundo da saúde 2005; 29(1):20-25.

23. Cohn A, Elias PEM. Equidade e reformas na saúde nos anos 90. Cad Saude Publica 2002; 18(Supl.):173180.

Artigo apresentado em 08/05/2009

Aprovado em 08/07/2009

Versão final apresentada em 20/07/2009 